\title{
INTERVAL-VALUED FUZZY IRRESOLUTE MAPPINGS ON INTERVAL-VALUED FUZZY TOPOLOGICAL SPACES
}

\author{
Won Keun Min and Myeong Hwan Kim
}

\begin{abstract}
We introduce the concepts of IVF irresolute mappings and IVF irresolute open mappings, and investigate characterizations for such mappings on the interval-valued fuzzy topological spaces.
\end{abstract}

\section{Introduction}

Zadeh [4] introduced the concept of fuzzy set and investigated basic properties. Gorzalczany [1] introduced the concept of interval-valued fuzzy set which is a generalization of fuzzy sets. In [3], Mondal and Samanta introduced the concepts of interval-valued fuzzy topology, continuity and compactness and studied some topological properties. In [2], Jun et al. introduced the concepts of IVF semiopen sets and IVF semiopen mappings and studied some results about them.

In this paper, we introduce the concepts of IVF irresolute mappings and IVF irresolute open mappings, and investigate characterizations for such mappings.

\section{Preliminaries}

Let $D[0,1]$ be the set of all closed subintervals of the interval $[0,1]$. The elements of $D[0,1]$ are generally denoted by capital letters $M, N, \cdots$ and note that $M=\left[M^{L}, M^{U}\right]$, where $M^{L}$ and $M^{U}$ are the lower and the

Received January 05, 2010. Accepted November 22, 2010.

Key words and phrases: IVF irresolute, IVF irresolute open, IVF semicontinuous, IVF continuous.

Corresponding author:wkmin@kangwon.ac.kr. 
upper end points respectively. Especially, we denote $\mathbf{0}=[0,0], \mathbf{1}=[1,1]$, and $\mathbf{a}=[a, a]$ for $a \in(0,1)$. We also note that

(1) $(\forall M, N \in D[0,1])\left(M=N \Leftrightarrow M^{L}=N^{L}, M^{U}=N^{U}\right)$.

(2) $(\forall M, N \in D[0,1])\left(M \leq N \Leftrightarrow M^{L} \leq N^{L}, M^{U} \leq N^{U}\right)$.

For every $M \in D[0,1]$, the complement of $M$, denoted by $M^{c}$, is defined by $M^{c}=\mathbf{1}-M=\left[1-M^{U}, 1-M^{L}\right]$. Let $X$ be a nonempty set.

A mapping $A: X \rightarrow D[0,1]$ is called an interval-valued fuzzy set (simply, IVF set) in $X$. For each $x \in X, A(x)$ is a closed interval whose lower and upper end points are denoted by $A(x)^{L}$ and $A(x)^{U}$, respectively. For any $[a, b] \in D[0,1]$, the IVF set whose value is the interval $[a, b]$ for all $x \in X$ is denoted by $\widetilde{[a, b]}$. In particular, for any $c \in[a, b]$, the IVF set whose value is $\mathbf{c}=[c, c]$ for all $x X$ is denoted by simply $\widetilde{c}$. For a point $p \in X$ and for $[a, b] \in D[0,1]$ with $b>0$, the IVF set which takes the value $[a, b]$ at $p$ and $\mathbf{0}$ elsewhere in $X$ is called an interval-valued fuzzy point (simply, IVF point) and is denoted by $[a, b]_{p}$. In particular, if $b=a$, then it is also denoted by $a_{p}$. Denoted by $D^{X}$ the set of all IVF sets in $X$.

For every $A, B \in D^{X}$, we define

$$
\begin{aligned}
& A=B \Leftrightarrow(\forall x \in X)\left([A(x)]^{L}=[B(x)]^{L} \text { and }[A(x)]^{U}=[B(x)]^{U}\right), \\
& A \subseteq B \Leftrightarrow(\forall x \in X)\left([A(x)]^{L} \subseteq[B(x)]^{L} \text { and }[A(x)]^{U} \subseteq[B(x)]^{U}\right) .
\end{aligned}
$$

The complement $A^{c}$ of $A$ is defined by

$$
\left[A^{c}(x)\right]^{L}=1-[A(x)]^{U} \text { and }\left[A^{c}(x)\right]^{U}=1-[A(x)]^{L}
$$

for all $x \in X$.

For a family of IVF sets $\left\{A_{i}: i \in J\right\}$ where $J$ is an index set, the union $G=\cup_{i \in J} A_{i}$ and $F=\cap_{i \in J} A_{i}$ are defined by

$$
(\forall x \in X)\left([G(x)]^{L}=\sup _{i \in J}\left[A_{i}(x)\right]^{L},[G(x)]^{U}=\sup _{i \in J}\left[A_{i}(x)\right]^{U}\right),
$$

$(\forall x \in X)\left([F(x)]^{L}=\inf _{i \in J}\left[A_{i}(x)\right]^{L},[F(x)]^{U}=\inf _{i \in J}\left[A_{i}(x)\right]^{U}\right)$, respectively.

Let $f: X \rightarrow Y$ be a mapping and let $A$ be an IVF set in $X$. Then the image of $A$ under $f$, denoted by $f(A)$, is defined as follows

$$
\begin{aligned}
& {[f(A)(y)]^{L}= \begin{cases}\sup _{f(x)=y}[A(x)]^{L}, & \text { if } f^{-1}(y) \neq \emptyset, y \in Y \\
0, & \text { otherwise },\end{cases} } \\
& {[f(A)(y)]^{U}= \begin{cases}\sup _{f(x)=y}[A(x)]^{U}, & \text { if } f^{-1}(y) \neq \emptyset, y \in Y \\
0, & \text { otherwise },\end{cases} }
\end{aligned}
$$


for all $y \in Y$.

Let $B$ be an IVF set in $Y$. Then the inverse image of $B$ under $f$, denoted by $f^{-1}(B)$, is defined as follows

$$
(\forall x \in X)\left(\left[f^{-1}(B)(x)\right]^{L}=[B(f(x))]^{L},\left[f^{-1}(B)(x)\right]^{U}=[B(f(x))]^{U}\right) .
$$

Definition 2.1 ([3]). A family $\tau$ of IVF sets in $X$ is called an interval-valued fuzzy topology (simply, IVFT) on $X$ if it satisfies:

(1) $\mathbf{0 , 1} \in \tau$.

(2) $A, B \in \tau \Rightarrow A \cap B \in \tau$.

(3) For $i \in J, A_{i} \in \tau \Rightarrow \cup_{i \in J} A_{i} \in \tau$.

Every member of $\tau$ is called an IVF open set. An IVF set $A$ is called an IVF closed set if the complement of $A$ is an IVF open set. And $(X, \tau)$ is called an interval-valued fuzzy topological space (simply, IVFTS).

In an IVFTS $(X, \tau)$, for an IVF set $A$ in $X$, the IVF closure and the IVF interior of $A$, denoted by $\operatorname{cl}(A)$ and $\operatorname{int}(A)$, respectively, are defined as

$$
\begin{aligned}
& c l(A)=\cap\left\{B \in D^{X}: B^{c} \in \tau \text { and } A \subseteq B\right\}, \\
& \operatorname{int}(A)=\cup\left\{B \in D^{X}: B \in \tau \text { and } B \subseteq A\right\},
\end{aligned}
$$

respectively [3].

Definition $2.2([2])$. Let $A$ be an IVF set in an IVFTS $(X, \tau)$. Then $A$ is said to be $I V F$ semiopen if $A \subseteq \operatorname{cl}(\operatorname{int}(A))$. $A$ is said to be $I V F$ semiclosed if the complement of $A$ is IVF semiopen. Denote the set of all IVF semiopen (resp., IVF semiclosed) sets by $I V F S O(X)$ (resp., $\operatorname{IVFSC}(X))$.

Definition $2.3([2,3])$. Let $\left(X, \tau_{1}\right)$ and $\left(Y, \tau_{2}\right)$ be two IVFTS's. Then $f: X \rightarrow Y$ is said to be continuous (resp., IVF semicontinuous) if for every $B \in \tau_{2}, f^{-1}(B)$ is IVF open (resp., IVF semiopen).

Definition $2.4([2])$. Let $\left(X, \tau_{1}\right)$ and $\left(Y, \tau_{2}\right)$ be two IVFTS's. Then $f: X \rightarrow Y$ is said to be IVF open (resp., IVF semiopen) if for every $A \in \tau_{1}, f(A)$ is IVF open (resp., IVF semiopen). 


\section{IVF Irresolute Mappings}

Definition 3.1. Let $f: X \rightarrow Y$ be a mapping between IVFTS's $\left(X, \tau_{1}\right)$ and $\left(Y, \tau_{2}\right)$. Then $f$ is said to be IVF irresolute if for every IVF semiopen set $U$ of $Y, f^{-1}(U)$ is IVF semiopen.

Every IVF irresolute mapping is IVF semicontinuous mapping but the converse need not be true as seen in the next example.

Example 3.2. Let $X=[0,1]$ and let $A, B$ and $C$ be IVF sets defined as follows

$$
\begin{aligned}
& A(x)= \begin{cases}{\left[\frac{1}{3} x, \frac{2}{3} x\right],} & 0 \leq x \leq \frac{1}{2}, \\
{\left[-\frac{1}{2} x+\frac{5}{12},-\frac{2}{3} x+\frac{2}{3}\right],} & \frac{1}{2} \leq x \leq \frac{5}{6}, \\
{\left[0,-\frac{2}{3} x+\frac{2}{3}\right],} & \frac{5}{6} \leq x \leq 1\end{cases} \\
& B(x)=\left[\frac{1}{3} x, \frac{1}{2}\right], 0 \leq x \leq 1 \text {; } \\
& C(x)= \begin{cases}{\left[\frac{5}{6} x+\frac{1}{6}, x+\frac{1}{6}\right],} & 0 \leq x \leq \frac{5}{6}, \\
{\left[\frac{5}{6} x+\frac{1}{6}, 1\right],} & \frac{5}{6} \leq x \leq 1 .\end{cases}
\end{aligned}
$$

Define IVF topologies $\tau_{1}$ and $\tau_{2}$ on $X$ as follows

$$
\tau_{1}=\{\mathbf{0}, A, B, \mathbf{1}\} ; \quad \tau_{2}=\{\mathbf{0}, A, \mathbf{1}\}
$$

Note that the IVF set $C$ is IVF semiopen in $\left(X, \tau_{2}\right)$ but it is not an IVF semiopen set in the IVFTS $\left(X, \tau_{1}\right)$. Hence we know that obviously the identity mapping $f:\left(X, \tau_{1}\right) \rightarrow\left(X, \tau_{2}\right)$ is an IVF semicontinuous mapping but it is not IVF irresolute.

IVF continuous $\Rightarrow$ IVF semicontinuous $\Leftarrow$ IVF irresolute

In an IVFTS $(X, \tau)$, for an IVF set $A$ in $X$, the IVF semi-closure and the IVF semi-interior of $A$, denoted by $\operatorname{scl}(A)$ and $\operatorname{sint}(A)$, respectively, are defined as

$$
\begin{aligned}
& \operatorname{scl}(A)=\cap\left\{B \in D^{X}: B^{c} \in I V F S C(X) \text { and } A \subseteq B\right\} ; \\
& \operatorname{sint}(A)=\cup\left\{B \in D^{X}: B \in I V F S O(X) \text { and } B \subseteq A\right\} .
\end{aligned}
$$

Lemma 3.3. Let $(X, \tau)$ be an IVFTS and $A \in D^{X}$.

(1) $\mathbf{1}-\operatorname{sint}(A)=\operatorname{scl}(\mathbf{1}-A)$.

(2) $\mathbf{1}-\operatorname{scl}(A)=\operatorname{sint}(\mathbf{1}-A)$. 
Theorem 3.4. Let $f: X \rightarrow Y$ be a mapping between IVFTS's $\left(X, \tau_{1}\right)$ and $\left(Y, \tau_{2}\right)$. Then the following statements are equivalent:

(1) $f$ is IVF irresolute.

(2) $f^{-1}(B)$ is IVF semiclosed for each IVF semiclosed set $B$ of $Y$.

(3) $f(\operatorname{scl}(A)) \subseteq \operatorname{scl}(f(A))$ for each $A \in D^{Y}$.

(4) $\operatorname{scl}\left(f^{-1}(B)\right) \subseteq f^{-1}(\operatorname{scl}(B))$ for each $B \in D^{Y}$.

(5) $f^{-1}(\operatorname{sint}(B)) \subseteq \operatorname{sint}\left(f^{-1}(B)\right)$ for each $B \in D^{Y}$.

Proof. (1) $\Leftrightarrow(2)$ From Definition 2.2, it is obvious.

$(2) \Rightarrow(3)$ Let $A$ be any IVF set in $X$. Since $s c l(f(A))$ is an IVF semiclosed set containing $f(A)$, by $(2), f^{-1}(\operatorname{scl}(f(A)))$ is IVF semiclosed and $A \subseteq f^{-1}(\operatorname{scl}(f(A)))$. So $\operatorname{scl}(A) \subseteq \operatorname{scl}\left(f^{-1}(\operatorname{scl}(f(A)))=f^{-1}(\operatorname{scl}(f(A)))\right.$. It implies $f(\operatorname{scl}(A)) \subseteq \operatorname{scl}(f(A))$.

$(3) \Rightarrow(4)$ Let $B$ be any IVF set in $Y$. From (3), it follows that $f\left(\operatorname{scl}\left(f^{-1}(B)\right)\right) \subseteq \operatorname{scl}\left(f\left(f^{-1}(B)\right)\right) \subseteq \operatorname{scl}(B)$. Hence $\operatorname{scl}\left(f^{-1}(B)\right) \subseteq$ $f^{-1}(\operatorname{scl}(B))$.

$(4) \Rightarrow(5)$ For any IVF set $B$ of $Y$, from (4), it follows

$$
\begin{aligned}
f^{-1}(\operatorname{sint}(B)) & =\mathbf{1}-\left(f^{-1}(\operatorname{scl}(\mathbf{1}-B))\right) \\
& \subseteq \mathbf{1}-\operatorname{scl}\left(f^{-1}(\mathbf{1}-B)\right) \\
& =\operatorname{sint}\left(f^{-1}(B)\right) .
\end{aligned}
$$

Hence $f^{-1}(\operatorname{sint}(B)) \subseteq \operatorname{sint}\left(f^{-1}(B)\right)$.

$(5) \Rightarrow(1)$ Let $V$ be any IVF semiopen set in $Y$. By (5),

$$
f^{-1}(V)=f^{-1}(\operatorname{sint}(V)) \subseteq \operatorname{sint}\left(f^{-1}(V)\right) .
$$

So $f^{-1}(V)$ is an IVF semiopen set, and hence $f$ is IVF irresolute.

Theorem 3.5. Let $f: X \rightarrow Y$ be a bijective mapping between IVFTS's $\left(X, \tau_{1}\right)$ and $\left(Y, \tau_{2}\right)$. Then $f$ is IVF irresolute if and only if $\operatorname{sint}(f(A)) \subseteq f(\operatorname{sint}(A))$ for each $A \in D^{X}$.

Proof. Let $f$ be IVF irresolute. Then for any IVF set $A$ of $X$, $f^{-1}(\operatorname{sint}(f(A)))$ is IVF semiopen. From Theorem 3.4 and injectivity of $f$, it follows $f^{-1}(\operatorname{sint}(f(A))) \subseteq \operatorname{sint}\left(f^{-1}(f(A))\right)=\operatorname{sint}(A)$. Since $f$ is surjective, $\operatorname{sint}(f(A))=f\left(f^{-1}(\operatorname{sint}(f(A)))\right) \subseteq f(\operatorname{sint}(A))$.

For the converse, let $B$ be any IVF semiopen set of $Y$. From hypothesis and surjectivity of $f$, it follows

$$
f\left(\operatorname{sint}\left(f^{-1}(B)\right)\right) \supseteq \operatorname{sint}\left(f\left(f^{-1}(B)\right)\right)=\operatorname{sint}(B)=B .
$$


Since $f$ is injective, $\left.\operatorname{sint}\left(f^{-1}(B)\right)\right) \supseteq f^{-1}(B)$, and so $f^{-1}(B)$ is IVF semiopen. Hence $f$ is IVF irresolute.

Definition 3.6. Let $(X, \tau)$ be an IVFTS. An IVF set $A$ in $X$ is said to be IVF semicompact if for every IVF semiopen cover $\mathcal{A}=\left\{A_{i} \in\right.$ $\left.D^{X}: A_{i} \in \tau, i \in J\right\}$ of $A$, there exists $J_{0}=\{1,2, \cdots, n\} \subseteq J$ such that $A \subseteq \cup_{j \in J_{0}} A_{j}$.

Theorem 3.7. Let $f:\left(X, \tau_{1}\right) \rightarrow\left(Y, \tau_{2}\right)$ be IVF irresolute on two IVFTS's. If $A$ is an IVF semicompact set, then $f(A)$ is also IVF semicompact.

Proof. Let $\left\{B_{i} \in D^{Y}: B_{i} \in \tau_{2}, i \in J\right\}$ be an IVF semiopen cover of $f(A)$ in $Y$. Then $\left\{f^{-1}\left(B_{i}\right): i \in J\right\}$ is an IVF semiopen cover of $A$ in $X$. By definition of IVF semicompactness, there exists $J_{0}=\{1,2, \cdots, n\} \subseteq$ $J$ such that $A \subseteq \cup_{j \in J_{0}}\left(f^{-1}\left(B_{j}\right)\right)$. So

$$
\begin{aligned}
f(A) & \subseteq f\left(\cup_{j \in J_{0}}\left(f^{-1}\left(B_{j}\right)\right)\right) \\
& =\cup_{j \in J_{0}} f\left(f^{-1}\left(B_{j}\right)\right) \\
& \subseteq \cup_{j \in J_{0}} B_{j} .
\end{aligned}
$$

Hence $f(A)$ is IVF semicompact.

An IVF set $A$ in an IVF topological space $X$ is said to be $I V F$ compact [3] if every IVF open cover $\mathcal{A}=\left\{A_{i}: i \in J\right\}$ of $A$ has a finite IVF subcover.

Theorem 3.8. Let $f:\left(X, \tau_{1}\right) \rightarrow\left(Y, \tau_{2}\right)$ be IVF semicontinuous on two IVFTS's. If $A$ is an IVF semicompact set, then $f(A)$ is IVF compact.

Proof. It is easily proved from the definition of IVF semicontinuity and Theorem 3.7.

Definition 3.9. Let $\left(X, \tau_{1}\right)$ and $\left(Y, \tau_{2}\right)$ be two IVFTS's. Then $f$ : $X \rightarrow Y$ is called an IVF irresolute open (resp., IVF irresolute closed mapping if for every IVF semiopen (resp., IVF semiclosed) set $A$ of $X$, $f(A)$ is IVF semiopen (resp., IVF semiclosed) in $Y$.

Every IVF irresolute open (resp., IVF irresolute closed) mapping is IVF semiopen (resp., IVF semiclosed) but the converse need not be true.

Example 3.10. Consider the identity mapping $f:\left(X, \tau_{2}\right) \rightarrow\left(X, \tau_{1}\right)$ in Example 3.2. Then $f$ is an IVF semiopen mapping but not IVF irresolute open. 
Theorem 3.11. Let $f: X \rightarrow Y$ be a mapping on IVFTS's $\left(X, \tau_{1}\right)$ and $\left(Y, \tau_{2}\right)$. The the following are equivalent:

(1) $f$ is IVF irresolute open.

(2) $f(\operatorname{sint}(A)) \subseteq \operatorname{sint}(f(A))$ for $A \in D^{X}$.

(3) $\operatorname{sint}\left(f^{-1}(B)\right) \subseteq f^{-1}(\operatorname{sint}(B))$ for $B \in D^{Y}$.

(4) For $B \in D^{Y}$ and each IVF semiclosed set $A$ of $X$ with $f^{-1}(B) \subseteq$ $A$, there exists an IVF semiclosed set $C$ of $Y$ such that $B \subseteq C$ and $f^{-1}(C) \subseteq A$.

Proof. (1) $\Rightarrow(2)$ For $A \in D^{X}$,

$$
\begin{aligned}
f(\operatorname{sint}(A)) & =f\left(\cup\left\{B \in D^{X}: B \subseteq A, B \in I V F S O(X)\right\}\right) \\
& =\cup\left\{f(B) \in D^{Y}: f(B) \subseteq f(A), f(B) \in I V F S O(Y)\right\} \\
& \subseteq \cup\left\{U \in D^{Y}: U \subseteq f(A), U \in I V F S O(Y)\right\} \\
& =\operatorname{sint}(f(A)) .
\end{aligned}
$$

Hence $f(\operatorname{sint}(A)) \subseteq \operatorname{sint}(f(A))$.

$(2) \Rightarrow(3)$ For $B \in D^{Y}$, from (2), we have

$$
f\left(\operatorname{sint}\left(f^{-1}(B)\right)\right) \subseteq \operatorname{sint}\left(f\left(f^{-1}(B)\right)\right) \subseteq \operatorname{sint}(B) .
$$

Hence $\operatorname{sint}\left(f^{-1}(B)\right) \subseteq f^{-1}(\operatorname{sint}(B))$.

$(3) \Rightarrow(4)$ Let $A$ be an IVF semiclosed set of $X$ with $f^{-1}(B) \subseteq A$ for $B \in D^{Y}$. Since $\mathbf{1}-A \subseteq \mathbf{1}-f^{-1}(B)=f^{-1}(\mathbf{1}-B)$ and $\operatorname{sint}(\mathbf{1}-$ $A)=\mathbf{1}-A \subseteq \operatorname{sint}\left(f^{-1}(\mathbf{1}-B)\right)$. By $(3), \mathbf{1}-A \subseteq \operatorname{sint}\left(f^{-1}(\mathbf{1}-B)\right) \subseteq$ $f^{-1}(\operatorname{sint}(\mathbf{1}-B))$. Thus $A \supseteq \mathbf{1}-\left(f^{-1}(\operatorname{sint}(\mathbf{1}-B))\right)=f^{-1}(\mathbf{1}-\operatorname{sint}(\mathbf{1}-$ $B))=f^{-1}(\operatorname{scl}(B))$. Now set $C=\operatorname{scl}(B)$. Then $C$ is an IVF semiclosed set of $Y$ such that $B \subseteq C$ and $f^{-1}(C) \subseteq A$.

$(4) \Rightarrow(1)$ Let $A$ be an IVF semiopen set of $X$. Then $f^{-1}(\mathbf{1}-f(A))=$ $\mathbf{1}-f^{-1}(f(A)) \subseteq \mathbf{1}-A$ and $\mathbf{1}-A$ is IVF semiclosed. By (4), there exists an IVF semiclosed set $C \in D^{Y}$ such that $\mathbf{1}-f(A) \subseteq C$ and $f^{-1}(C) \subseteq \mathbf{1}-A$. It implies $\mathbf{1}-C \subseteq f(A)$ and $f(A) \subseteq f\left(\mathbf{1}-f^{-1}(C)\right)=$ $f\left(f^{-1}(\mathbf{1}-C)\right) \subseteq \mathbf{1}-C$.

Hence $f(A)$ is an IVF semiopen set in $Y$.

Theorem 3.12. Let $f: X \rightarrow Y$ be a mapping on IVFTS's $\left(X, \tau_{1}\right)$ and $\left(Y, \tau_{2}\right)$. The the following are equivalent:

(1) $f$ is IVF irresolute closed.

(2) $\operatorname{scl}(f(A)) \subseteq f(\operatorname{scl}(A))$ for $A \in D^{X}$. 


$$
\begin{aligned}
\text { Proof. (1) } & \Rightarrow(2) \text { For } A \in D^{X}, \\
f(\operatorname{scl}(A)) & =f\left(\cap\left\{B \in D^{X}: A \subseteq B, B \in I V F S C(X)\right\}\right) \\
& =\cap\left\{f(B) \in D^{Y}: f(A) \subseteq f(B), f(B) \in I V F S C(Y)\right\} \\
& \supseteq \cap\left\{F \in D^{Y}: f(A) \subseteq F, F \in I V F S C(Y)\right\} \\
& =\operatorname{scl}(f(A)) .
\end{aligned}
$$

Hence $\operatorname{scl}(f(A)) \subseteq f(\operatorname{scl}(A))$.

$(2) \Rightarrow(1)$ Let $A$ be an IVF semiclosed set in $X$. Then by (2),

$$
\operatorname{scl}(f(A)) \subseteq f(\operatorname{scl}(A))=f(A) .
$$

It implies $f(A)$ is IVF semiclosed, and so $f$ is IVF irresolute closed.

Theorem 3.13. Let $f: X \rightarrow Y$ be a bijective mapping between IVFTS's $\left(X, \tau_{1}\right)$ and $\left(Y, \tau_{2}\right)$. Then

(1) $f$ is IVF irresolute closed.

(2) $\operatorname{scl}(f(A)) \subseteq f(\operatorname{scl}(A))$ for $A \in D^{X}$.

(3) $f^{-1}(\operatorname{scl}(B)) \subseteq \operatorname{scl}\left(f^{-1}(B)\right)$ for each $B \in D^{Y}$.

Proof. It is sufficient to show that (2) is equivalent to (3).

$(2) \Rightarrow(3)$ For $B \in D^{Y}$, since $f$ is surjective,

$$
\operatorname{scl}(B)=\operatorname{scl}\left(f\left(f^{-1}(B)\right)\right) \subseteq f\left(\operatorname{scl}\left(f^{-1}(B)\right)\right) .
$$

From injectivity of $f$,

$$
f^{-1}(\operatorname{scl}(B)) \subseteq f^{-1}\left(f\left(\operatorname{scl}\left(f^{-1}(B)\right)\right)\right)=\operatorname{scl}\left(f^{-1}(B)\right) .
$$

$(3) \Rightarrow(2)$ Conversely, let $A \in D^{X}$. Then from hypothesis and injectivity of $f, f^{-1}(\operatorname{scl}(f(A))) \subseteq \operatorname{scl}\left(f^{-1}(f(A))\right)=\operatorname{scl}(A)$ Since $f$ is surjective, $f(\operatorname{scl}(A)) \supseteq f\left(f^{-1}(\operatorname{scl}(f(A)))\right)=\operatorname{scl}(f(A))$. Hence the statement (2) is obtained.

\section{References}

[1] M. B. Gorzalczany, A method of inference in approximate reasoning based on interval-valued fuzzy sets, J. Fuzzy Math. 21 (1987), 1-17.

[2] Y. B. Jun, G. C. Kang and M. A. Ozturk Interval-valued fuzzy semiopen, preopen and $\alpha$-open mappings, Honam Math. J., 28(2) (2006), 241-259.

[3] T. K. Mondal and S. K. Samanta, Topology of interval-valued fuzzy sets, Indian J. Pure Appl. Math., 30(1) (1999), 23-38.

[4] L. A. Zadeh, Fuzzy sets, Information and Control 8 (1965), 338-353. 
Department of Mathematics,

Kangwon National University,

Chuncheon, 200-701, Korea

E-mail: wkmin@kangwon.ac.kr

E-mail: mhkim@kangwon.ac.kr 